DOI: $10.1002 /$ mabi.201100341

Article type: Full Paper

\title{
Controlled cell proliferation on an electrochemically engineered collagen scaffold
}

Robert Gendron*, M. Ramesh Kumar, Helene Paradis*, Darryl Martin, Nhu Ho, Danielle Gardiner, Erika F. Merschrod S.*, Kristin M. Poduska*

Prof. R. Gendron, Prof. H. Paradis, N. Ho, D. Gardiner

Division of Biomedical Sciences, Faculty of Medicine

Memorial University, St. John's, NL, A1B 3V6 (Canada)

E-mail: rgendron@mun.ca, hparadis@mun.ca

M. R. Kumar, Prof. E. F. Merschrod S, Prof. K. M. Poduska

Department of Chemistry

Memorial University, St. John's, NL, A1B 3X7 (Canada)

E-mail: erika@mun.ca, kris@mun.ca

Dr. D. Martin

Department of Surgery, Section of Urology,

Yale University School of Medicine, New Haven, CT (USA)

Therapies for corneal disease and injury often rely on artificial implants, but integrating cells into synthetic corneal materials remains a significant challenge. Formed using electrochemical techniques to manipulate protein aggregation, a collagen-based material shows promise as a scaffold for corneal repair. There is histological and molecular evidence for a specific interaction of corneal fibroblasts with this novel transparent matrix scaffold material. Most significantly, the electrochemically produced matrix is non-toxic to cells and controls the proliferation in the corneal fibroblasts seeded onto it. This is similar to the behavior of corneal stromal cells in a native corneal environment. Not only is this result is an important first step toward developing a more realistic, multi-component artificial cornea, but it also opens possibilities for using this matrix to control and contain the growth of cells in engineered tissues. 


\section{Introduction}

The corneal stroma, containing keratocytes and a range of extracellular matrix molecules, contributes to a highly specialized microenvironment that maintains healthy vision. Disease and injury to the cornea can lead to unhindered cell proliferation, tissue dysregulation, or loss of its optical transparency. A corneal transplant (keratoplasty) is a possible remedy when severe degradation occurs, but there are significant risks such as graft rejection. ${ }^{[1]}$ As an alternative, corneal repair could also be facilitated by replacing damaged or diseased tissue with a scaffold that promotes appropriate regeneration by either native (autologous) or donor (homologous) corneal fibroblast cells. ${ }^{[2]}$ To be medically viable, artificial cornea materials should support corneal cell activity without instigating the kind of uncontrolled growth that occurs in tumors. In short, the material must provide the correct chemical, structural and mechanical environment to the cells in order to regulate their response.

Biocompatible matrix scaffolds are widely studied, ${ }^{[3-5]}$ and there are numerous commercial matrices that support and/or stimulate cell growth (including Matrigel and fibronectin glue). Many of these matrices are naturally derived composite materials that involve different kinds of proteins and growth factors that promote cell attachment and growth. ${ }^{[4]}$ In vitro studies have shown that cell attachment can be guided through topographical, chemical, or mechanical cues. ${ }^{[5]}$ However, the mechanisms behind cell attachment and growth at different kinds of surfaces remains a complex and discipline-wide question. Artificial scaffolds used in corneal implants can also suffer from issues such as lack of transparency and discoloration, as well as mechanical failures. ${ }^{[2]}$

For cell growth studies, immortalized cell lines are often convenient. Unlike normal cells that eventually cease division once chromosomal DNA becomes damaged, immortalized cell lines (including cancer and stem cells) are designed to divide indefinitely. In the context of in vitro cell culture studies, immortalized - but contact-inhibited - cells slow their 
proliferation rates when they run out of space. Conversely, immortalized and transformed cells continue to proliferate on top of each other when they run out of surface space. In vivo, unchecked cell growth is the hallmark of transformed cancerous cells. Thus, it is advantageous to identify cell culture matrices that encourage contact inhibition and do not support cell transformation.

Different cell lines do not necessarily respond the same way to a given matrix, so it is important to test cell viability using application-appropriate lines. Gendron et al. derived an immortalized cell line, MK/T-1, from mouse corneal stromal cultures. ${ }^{[6]}$ These cells are not senescent and do not exhibit a transformed phenotype; in other words, these cells continue to divide but do not lose control of their proliferation. In this regard, MK/T-1 cells are very unique because they are the only reported existing immortalized cell line that recapitulates the corneal fibroblast phenotype without a cancerous (oncogenic) transformation. These cells retain properties of specific phenotypic stages of corneal fibroblasts: they upregulate expression of smooth muscle alpha-actin (ASMA), a marker of myofibroblastic transdifferentiation, and activate the p38 mitogen-activated protein Kinase (p38 MAPK) pathway upon transforming growth factor beta (TGF-beta) induction. ${ }^{[7]}$ Furthermore, MK/T1 cells have served several research groups as a useful model for corneal stromal cell biology. ${ }^{[8]}$

This in vitro study demonstrates a successful first step toward potential therapeutic, cornea-specific applications of a scaffold material that limits the growth of an immortalized cell line without killing the cells.

\section{Experimental Section}

\section{Matrix scaffolds}


An electrochemically induced collagen aggregation procedure, based on isoelectric focusing between parallel plate electrodes, was applied to an electrolyte containing type I collagen monomers $(0.07 \mathrm{mg} / \mathrm{mL}$ from $3 \mathrm{mg} / \mathrm{mL}$ Vitrogen stock solution, Inamed Biomaterials) in ultrapure water $(18.2 \mathrm{M} \Omega \bullet \mathrm{cm}$, Barnstead Nanopure) adjusted to $\mathrm{pH} 7$ with $\mathrm{NaOH}$ (EMD chemicals, ACS reagent grade) [9]. A potential (8 V for 30 minutes) applied to this electrolyte caused the formation of a transparent membrane (thickness $\sim 500 \mu \mathrm{m}$, with surface area $\sim 1$ $\mathrm{cm}$ x $2.5 \mathrm{~cm})$. Membranes were placed immediately on sterile culture dishes and refrigerated until cell culture studies commenced. Parallel sets of membranes were prepared for materials characterization. They were dried onto glass slides for atomic force microscopy and force spectroscopy (Asylum Research MFP-3D), Raman spectroscopy (Jobin Yvon Horiba LabRAM, confocal, $532 \mathrm{~nm}$ excitation), and Scanning Electron Microscopy (SEM) investigations (FEI Quanta 400 environmental SEM, with carbon-coated specimens). Wet membranes were used for ultraviolet (UV)-visible absorption spectroscopy (OceanOptics USB2000).

Control thermally fibrilized collagen specimens were prepared by fibrilizing collagen by incubating the same monomeric starting collagen $(0.1 \mathrm{mg} / \mathrm{mL}$ from $3 \mathrm{mg} / \mathrm{mL}$ Vitrogen stock solution, Inamed Biomaterials) in a phosphate buffer at $\mathrm{pH} 6$ at $37^{\circ} \mathrm{C}$ for 4 hours. Additional control experiments were performed in standard polystyrene tissue culture plates with no collagen present.

\section{Cell Cultures}

Standard polystyrene tissue culture plates holding the collagen matrix were UV-sterilized (10 minutes, $30 \mathrm{~W} / 3 \mathrm{~A}$ bulb) in a tissue culture laminar flow cabinet, then washed with Dulbecco's Modified Eagle Medium (DMEM, 3 times for 5 minutes). MK/T-1 cells were trypsinized, washed, counted and then replated onto the scaffold coated plates $\left(0.17 \times 10^{6}\right.$ cells per $21 \mathrm{~cm}^{2}$ ) or simultaneously and at the same density onto the same type of standard untreated tissue culture plates in DMEM with fetal bovine serum (FBS, 10\%). Cells were 
allowed to gently settle onto the matrix and/or the untreated tissue culture plates before standard tissue culture incubation $\left(37^{\circ} \mathrm{C}\right.$, humidified $5 \% \mathrm{CO}_{2}$ atmosphere) to maintain exponential growth conditions. Some experiments required cultures incubated with 5-bromo2-deoxyuridine $\left(\mathrm{BrdU}, 1\right.$ hour at $\left.37^{\circ} \mathrm{C}\right)$. Cultures were maintained for 48 hours before harvesting.

\section{Histology}

Cell/scaffold co-cultures and plastic replated cultures were gently scraped from the dishes after 48 hours of culture and centrifuged (1000 rpm for 5 minutes) to gently pellet the cells and matrix. Pellets were fixed in phosphate buffered saline with paraformaldehyde (4\%) for 48 hours before embedding in a low-melting-temperature agarose and subsequent embedding in paraffin blocks so that $5 \mu \mathrm{m}$ serial sections could be generated, numbered and registered. Hematoxylin and eosin (H\&E) staining on every 20th section mapped the location and integrity of cell-matrix materials. Sections identified by H\&E mapping were used for immunohistochemistry analysis of gene expression. To ensure that differences in expression of PCNA and BrdU staining are meaningful (and not due to differences in cell integrity), sections adjacent to those used for PCNA/BrdU studies were treated with histological H\&E stain. H\&E staining indicates a sufficient number of cells for both types of cultures, and evidence of robust $\alpha$-tubulin protein expression in matrix-cultured cells provides a further positive control for cellular integrity and protein expression integrity in matrix cultures. Tubulin is a cytoskeletal scaffolding protein that plays a critical role in processes such as cell division.

\section{Antibodies}

Antibodies used in this study include rabbit anti-phospho-p44/42 MAPK (Thr202/Tyr204, New England Biolabs, Beverly MA, used at 1/2000 for western blotting and 1/250 for immunohistochemistry), rabbit anti-p44/42 MAPK (Santa Cruz Biotechnology, used at dilutions of 1/1000 for western and 1/250 for immunohistochemistry), rabbit anti-PCNA (FL- 
261, Santa Cruz Biotechnology, Inc. USA, used at 1/2000 for western blotting and 1/100 for immunohistochemistry), mouse monoclonal DM1 anti-Tubulin (Sigma, St. Louis MI, used at $1 \mu \mathrm{g} / \mathrm{ml}$ for western and 1/250 dilution for immunohistochemistry), mouse monoclonal 1A4 anti-ASMA (Sigma, St. Louis MI, used at 1/6500 for western and 1/200 for immunohistochemistry) and mouse monoclonal anti-BrdU (Sigma, St. Louis MI, used at 1:100 for immunohistochemistry). Appropriate chromogen conjugated secondary antibody reagents (Promega, Madison WI) were used to develop the reactions.

\section{Immunohistochemistry}

Immunohistochemical staining was performed sections that were deparaffinized in xylene and graded ethanols and then held in Tris buffered saline (TBS). Sections were blocked in ECL Advance Blocking Agent (GE Healthcare, Buckinghamshire UK, 2 hrs in 2\%, in TBS with 0.1\% Triton X-100 added for nuclear markers phospho-MAPK, PCNA and BrdU). After blotting the blocking solution, sections were treated with primary antibodies in ECL Advance Blocking Agent in TBS overnight. Sections were washed in TBS (3 times for 10 minutes) and then incubated in either anti-rabbit Ig Alkaline Phosphatase, anti-mouse Ig Alkaline Phosphatase or, in the case of tubulin, peroxidase (Promega, in 2\% ECL Advance Blocking Agent in TBS at 1/200 dilution for $~ 2$ hours). After washing in TBS (3 times for 10 minutes), sections were developed using Vector Red AP with Levamisole (Vector Laboratories, Burlingame CA USA) or Vector NovaRed (Vector Laboratories, Burlingame CA USA) for peroxidase conjugated secondary antibodies. Sections were then air dried and mounted with Permount.

\section{Western blot analysis}

Western blots were performed by standard procedures as previously described.[6] Cells were isolated from surrounding matrix scaffold by incubating the material in Versene EDTA solution $\left(37^{\circ} \mathrm{C}\right)$ with gentle pipetting, while cells which had been replated on standard tissue culture plastic could be gently scraped from the dishes. Chemiluminescence detection 
reagents (Amersham Biosciences, Piscataway, NJ) allowed measurement of ASMA, phosphop44/42 MAPK, p44/42 MAPK, PCNA using the antibody reagents described above for immunohistochemistry. ASMA Western blots were stripped and re-probed with anti- $\alpha$-tubulin monoclonal antibody to verify protein integrity and loading equivalency. Phospho-p44/42 MAPK Western blots were stripped and reprobed with anti-p44/42 MAPK and PCNA. Densitometry analyses were conducted using the Kodak Gel Logic Imaging System (Eastman Kodak Company, Rochester, NY) and intensities of the expressed bands were analyzed using Kodak Molecular Imaging Software (Version 4.0). ASMA and PCNA band intensities were compared to Tubulin band intensities as the loading control while p42 MAPK and p44 MAPK band intensities were used as loading controls for comparing the band intensities of the p42 and p44 phosphorylated forms of MAPK.

\section{Results and Discussion}

\section{Matrix properties}

The collagen matrix scaffold, generated using an electrochemically induced collagen aggregation procedure, displays physical properties such as optical transparency, mechanical stiffness, and water incorporation that are desirable for use in artificial ocular implants. ${ }^{[1]}$ The matrix formation process is based on isoelectric focusing between parallel plate electrodes, ${ }^{[9]}$ resulting in a sheet-like scaffold (Figure 1a) with a thickness $\sim 500 \mu \mathrm{m}$ when dried (as measured by AFM). When fully hydrated, the scaffold swells to a thickness of several millimeters and becomes softer and more viscous. This is the condition under which the cell culture studies were conducted since this is analogous to in vivo conditions. The lateral dimensions of the scaffold are determined by the size of the electrodes; those used for this study are $\sim 1 \mathrm{~cm}$ x $2.5 \mathrm{~cm}$. Previous studies have shown that these layers are comprised of a fibrillar collagen core with a monomeric collagen overlayer, based on morphological features in atomic force microscopy images and from characteristic amine-related peak positions in Raman spectroscopic data. ${ }^{[9]}$ Our matrix has high optical transparency in the visible range, 
with over $90 \%$ transmittance above $400 \mathrm{~nm}$ (Figure 1b). This is only slightly lower than the 95-99\% transmittance observed in natural corneas. ${ }^{[1]}$

The elastic modulus of the matrix can be controlled with the addition of different cations during membrane formation; ${ }^{[9]}$ the membranes used here have Young's elastic moduli of $0.10-0.22 \mathrm{GPa}$ when fully desiccated, as calculated from nanoindentation measurements. These are similar to values obtained by others for partially desiccated human corneas, which are considerably higher than values obtained on fully hydrated corneas, as expected. ${ }^{[10]} \mathrm{We}$ note that in vivo, scaffolds would exist in a fully hydrated state. These mechanical properties make the scaffolds quite robust during routine laboratory handling, and they can be dried and rehydrated multiple times without changing the mechanical stiffness of the membrane. ${ }^{[9]}$

We note that this electrochemical assembly method does not apply equally well to all types of proteins. For example, replacing collagen with either fibronectin or bovine serum albumin does not lead to mechanically stable membranes. Instead, the resulting products with these two other proteins were small, mm-sized globular aggregates that remained suspended in the electrolyte rather than aggregating together.

\section{Cell viability}

A biocompatibility test ensured that cells cultured in the presence of the matrix are viable. MK/T-1 cells that adhere to the electrochemically induced matrix scaffold appear robustly healthy, adhesive, motile, and display pseudopodia (Figure 2a). In addition, MK/T-1 cells that adhere to the matrix scaffold appear to retain the property of contact inhibition, since we did not observe the cells piling up on each other when in contact with our electrochemically induced matrix. This property is not due to a low cell concentration, but rather a property of the cell line itself, as described in earlier studies. ${ }^{[6]}$ The observed cell morphologies are similar to those when these cells are grown on surfaces designed to maintain viability, such as a tissue culture plastic control (Figure 2b). Hematoxylin and eosin 
(H\&E) stained paraffin embedded sections (Figure 2c,d) showed no signs of the fragmented or shrivelled nuclei that would be characteristic of programmed cell death (apoptosis or pyknosis). In stark contrast to the good cell adherence on the electrochemical scaffold and the plastic control, the fibrillized collagen control did not induce any specific cell positioning or flattening (Figure 2e). This indicates that the collagen treatment procedure prior to cell culturing can have a critical impact on the fundamental nature by which the scaffold can support viable cells.

\section{Cell activity}

A surprising and unique result of subsequent cell culture studies is that our electrochemically induced collagen matrix does not appear to stimulate the MK/T-1 cells into a more proliferative state, in which the cells would duplicate their DNA more actively. A granular nuclear staining pattern for the cell cycle marker proliferating cell nuclear antigen (PCNA) appears in the control plastic-replated cells and control thermally fibrillized collagen replated cells (Figure 3a,b). In contrast, the MK/T-1 cells display a diffuse nuclear pattern of expression when adhered to the electrochemically induced collagen scaffold (Figure 3c). Negative control immuno-staining reactions (shown in Figure 3d for plastic-replated cells) confirm the specificity of the anti-PCNA stain. Previous studies have shown that diffuse PCNA expression, such as that displayed by our electrochemically induced matrix-cultured cells, is observed in cells that are outside the DNA replication (S-phase) portion of the cell cycle, while granular nuclear PCNA expression coincides with cells in S-phase that are actively replicating their DNA. ${ }^{[1]}$ Although our investigations show that the spatial pattern of intracellular localization of PCNA in electrochemically induced matrix and plastic replated cells differ, the level of expression of PCNA by western blot analyses revealed no significant quantitative difference between the two conditions (Figure 4a). 
We assessed the DNA replication question more directly by replating the MK/T-1 cells in the presence of 5-bromo-2-deoxyuridine (BrdU, a synthetic analogue of thymidine) on both the electrochemically induced collagen scaffold and the control plastic replating conditons. High levels of BrdU inside the cells indicate active DNA replication, and its incorporation was measured by immunostaining. While plastic-replated control cells incubated with BrdU displayed robust BrdU staining (Figure 5a), the level of BrdU staining in nuclei of electrochemical matrix replated cultures (Figure 5b) was similar to background levels observed in negative control immunostaining (Figure 5c). It is important to note that, while the same numbers of cells were simultaneously replated onto either the plastic or the electrochemical matrix, the cells were ansynchronous populations, implying that our experiments sampled cells at different points in their life cycles. Since no observable fraction of the cells were actively replicating DNA as evidenced by the lack of BrdU stained cells, we conclude that cells on our electrochemically induced matrix appeared more quiescent than proliferative. This response is very different from the active DNA replication observed, based on the high levels of BrdU stained cells, when cells are replated onto the plastic control surface.

\section{Cell metabolism}

Quiescent cells are known to maintain an active metabolism, ${ }^{[12]}$ and we observed this in the electrochemically induced matrix cultures. Figure 6a,b shows prominent expression of a marker related to cell motility, alpha-smooth muscle actin (ASMA), and subsequent western blot analyses (Figure 4b) show that ASMA expression is slightly but significantly upregulated in matrix replated cultures compared to plastic replated cultures (average fold increase with standard error of mean $1.7+/-0.02$ ).

In addition, in electrochemically induced matrix replated MK/T-1 cells, we observe specific patterns of expression of activated intracellular signalling molecules that are involved 
in a number of processes such as cell growth and differentiation. The levels of an activated signalling cascade for an extracellular signal-regulated kinase (phospho-p42 mitogenactivated protein kinase, MAPK) was significantly upregulated in electrochemically induced matrix-replated cells compared to plastic-replated cells (average fold increase with standard error of mean $8.3+/-0.47$ ), as seen in Figure 4a and $6 \mathrm{c}$. On the other hand, levels of activated phospho-p44 MAPK (Erk1) were not significantly different in matrix-replated versus plastic replated MK/T-1 cells, as shown in Figure 4a.

These metabolic findings are significant because they suggest our electrochemically induced collagen matrix triggers differentiative rather than prolifative signaling in the MK/T1 cells. Earlier work demonstrates that MK/T-1 cells upregulate a similar signaling pathway (p38 MAPK) and ASMA expression when exposed to a transforming growth factor (TGF-b 2). ${ }^{[6]}$ Others have clearly shown that cells adhering to collagen can activate the p44/42 MAPK signaling pathway based on induction of phospho-p44/42 MAPK expression. ${ }^{[13]}$ Transient activation of the p44/42 MAPK signaling pathway has been previously associated with cell proliferation, while sustained activation, as we observe here in electrochemically induced matrix scaffold replated MK/T-1 cells, has been linked to differentiation. ${ }^{[14]}$ This earlier work also showed that the regulation of the kinetics of $\mathrm{p} 44 / 42$ MAPK activation is one of the mechanisms involved in the differentiation of neuronal cells. While this limited literature indicates the importance of sustained p44/42 MAPK activation in cell differentiative responses, we are not aware of any other studies showing specific activation of only p42 MAPK in a cell differentiative response to collagen as we observed here in the collagen matrix replated MK/T-1 cells. This exciting finding merits further study since it suggests that the electrochemically produced matrix scaffold might impart unique MAPK signalling properties to the cells it contacts.

\section{Matrix modifications}


Although it is not yet clear which specific attributes of our electrochemically produced collagen matrix enable it to stall the proliferation of the immortalized MK/T-1 cells, control experiments using collagen that was thermally fibrilized (with no electric field) suggest that the presence of the collagen itself does not quell cell proliferation. This is evident in the comparisons of PCNA pattern of expression described in Figure 3. The cells themselves also play a key role in the non-proliferation. In the electrochemically assembled matrix, there are clearly identifiable areas onto which MK/T-1 cells do not spread (Figure 2c, 3b, 6b,c,d). This suggests that cell-cell contact inhibition of the MK/T-1 cells cannot be the sole contributing factor to the apparent quiescence we observe. We note that others have used similar isoelectric focusing methods, but with a different electrode geometry, to produce aligned onedimensional collagen bundles rather than our two-dimensional collagenous sheets. ${ }^{[15]}$ Their cell growth studies, involving fibroblasts suited for tendon or ligament repair, demonstrate cell viability but do not address the possibility of quiescence. Thus, while the degree of collagen aggregation appears to be one factor affecting cell quiescence, there is more to learn about controlling these complex matrix-cell interactions.

\section{Conclusion}

Our cell-culture studies show that electrochemically prepared collagen matrices offer three important attributes for a synthetic cornea cell scaffold. First, the matrices display high optical transparency in the visible range, with over $90 \%$ transmittance above $400 \mathrm{~nm}$. Second, the matrix encourages cell attachment near its surface, which keeps the cells near the interface and may improve their reactivity. Third, the collagen matrices appear to stimulate signaling pathways in the cornea-specific MK/T-1 cells and support contact inhibition without overly stimulating cell proliferation. This scenario might also be advantageous in contexts beyond cornea applications where viable and metabolically active, yet quiescent, cellular coatings are required on artificial biomaterial surfaces. Thus, this scaffold does more than merely support viable cells; our evidence shows that there are significant differences in the way cells 
proliferate on our electrochemically assembled collagen scaffolds compared to collagenous scaffold material assembled by other methods. For these reasons, the MK/T-1 cellelectrochemically induced collagen matrix scaffold co-culture system is a novel and potentially useful model in which to study the interactions of mammalian cells on a new artificial biomaterial, which could be an important step toward better engineering of artificial tissues.

Acknowledgements: We thank Ewa Miskiewicz and Jacqueline Walker for expert technical assistance, and Jordon Keats for assistance in preparing the collagen scaffolds.

Received: ((will be filled in by the editorial staff)); Revised: ((will be filled in by the editorial staff)); Published online: DOI: 10.1002/ mabi.201100341

Keywords: biomedical applications; functional coatings; hierarchical structures; polymeric materials; tissue engineering

[1] a) J.R. Hassell JR, D.E. Birk, Exp. Eye Res. 2010, 91, 326-335. b) F.R. Ghosheh, F.A. Cremona, C.J. Rapuano, E.J Cohen, B.D. Ayres, K.M. Hammersmith, I.M. Raber, P.R. Laibson, Int. Ophthalmol. 2008, 28,147-153. c) D. Myung, P.E. Duhamel, J.R. Cochran, J. Noolandi, C.N. Ta, C.W. Frank, Biotechnol. Prog. 2008, 24, 735-741. d) M. Griffith, M. Hakim, S. Shimmura, M.A. Watsky, F. Li, D. Carlsson, C.J. Doillon, M. Nakamura, E. Suuronen, N. Shinozaki, K. Nakata, H. Sheardown, Cornea 2002, 21, S54-61. e) D.M. Maurice, J. Physiol. 1957, 136, 263-286.

[2] C.R. Hicks, G.J. Crawford, X. Lou, D.T. Tan, G.R. Snibson, G. Sutton, N. Downie, L Werner, T.V. Chirila, I.J. Constable, Eye 2003, 17, 385-392. 
[3] a) S. MacNeil, Nature 2007, 445, 874-880. b) Y.W. Fan, R.Z. Wang, Adv. Mater. 2005, 17, 2384-2388. c) H.Y. Li, L.A. Estroff, J. Am. Chem. Soc. 2007, 129, 5480-5483.

[4] a) G.A. Hudalla, W.L. Murphy, Adv. Funct. Mater. 2010, 21, 1754-1768. b) G. Wei, P.X. Ma, Adv. Funct. Mater. 2008, 18, 3568-3582. c) J.-T. Zhang, J. Nie, M. Mühlstädt, H.

Gallagher, O. Pullig, K.D. Jandt, Adv. Funct. Mater. 2011, Early view, DOI:

10.1002/adfm.201100739.

[5] a) L. Richert, F. Vetrone, J.-H. Yi, SF. Zalzal, J.D. Wuest, F. Rosei, A. Nanci, Adv. Mater. 2008, 20, 1488-1492. b) U. Cheema, C.-B. Chuo, P. Sarathchandra, S.N. Nazhat, R.A. Brown, Adv. Funct. Mater. 2007, 17, 2426-2431. c) C.S. Szot, C.F. Buchanan, P. Gatenholm, M.N. Rylander, J.W. Freeman, Mat. Sci. Eng. C 2011, 31, 37-42. d) D.E. Discher, P. Janmey, Y.-L. Wang, Science 2005, 310, 1139-1143.

[6] R.L. Gendron, C.Y. Liu, H. Paradis, L.C. Adams, W.W. Kao, Mol. Vis. 2001, 7, 107-113. [7] a) M.E. Fini, Prog. Retin. Eye Res. 1999, 18, 529-551. b) J.V. Jester, P.A. Barry-Lane, H.D. Cavanagh, W.M. Petroll, Cornea 1996, 15, 505-516.

[8] a) E.C. Carlson, K. Mamiya, C.Y. Liu, R.L. Gendron, D.E. Birk, J.L. Funderburgh, W.W. Kao, Biochem. J. 2003, 369, 461-468. b) M. Lin, E. Carlson, E. Diaconu, E. Pearlman, J. Leukoc. Biol. 2007, 81, 786-792.

[9] a) H.R. Baker, E.F. Merschrod S., K.M. Poduska, Langmuir 2008, 24, 2970-2972. b)

M.R. Kumar, E.F. Merschrod S., K.M. Poduska, Biomacromol. 2009, 10, 1970-1975.

[10] A.C. Jayasuriya, S. Ghosh, J.I. Scheinbeim, V. Lubkin, G. Bennett, P. Kramer, Biosens. Bioelectron. 2003, 18, 381-387.

[11] J.H. van Dierendonck, J.H. Wijsman, R. Keijzer, C.J. van de Velde, C.J. Cornelisse, Am. J. Pathol. 1991, 138, 1165-1172.

[12] S. Srivastava, R.K. Mishra, J. Dhawan, Organogenesis 2010, 6, 37-47.

[13] B.E. Slack, M.S. Siniaia, J. Cell Biochem. 2005, 95, 366-378. 
[14] A. von Kriegsheim, D. Baiocchi, M. Birtwistle, D. Sumpton, W. Bienvenut, N. Morrice, K. Yamada, A. Lamond, G. Kalna, R. Orton, D. Gilbert, W. Kolch. Cell Biol. 2009, 11, 14581464.

[15] X. Cheng, U.A. Gurkan, C.J. Dehen, M.P. Tate, H.W. Hillhouse, G.J. Simpson, O. Akkus. Biomater. 2008, 29, 3278-3288.
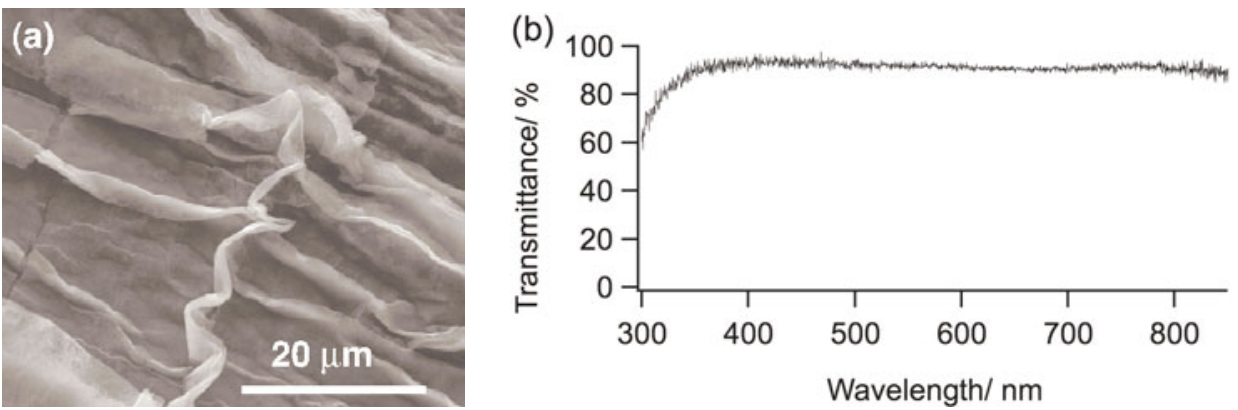

Figure 1. (a) A scanning electron micrograph highlights the sheet-like character of the electrochemically prepared collagen scaffolds. (b) A representative transmittance spectrum from a hydrated electrochemically prepared collagen matrix (prior to incubation with cells) demonstrates its high optical transparency throughout the visible region $(400-700 \mathrm{~nm})$. 

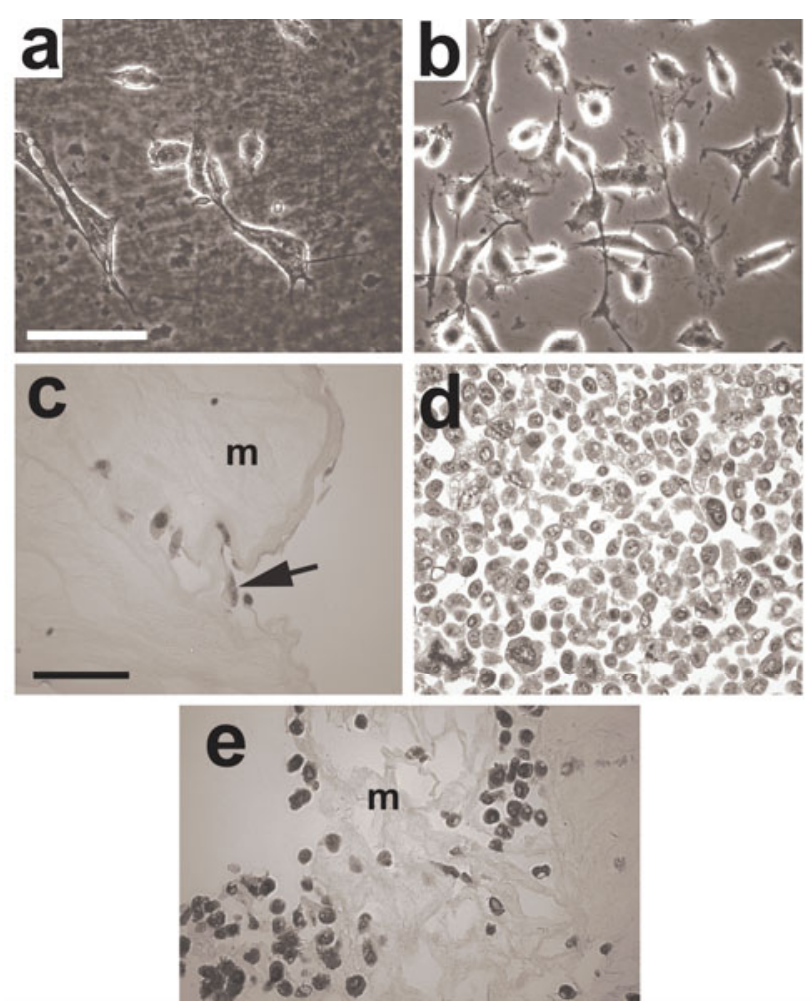

Figure 2. Phase-contrast optical microscopy $(\mathrm{a}, \mathrm{b})$ and H\&E stained paraffin sections of pelleted co-cultures $(\mathrm{c}, \mathrm{d}, \mathrm{e})$ show cell morphologies that indicate robust and healthy adherence and motility. For these images, cells were replated simultaneously onto the electrochemical collagen matrix scaffold $(a, c)$, tissue culture plastic $(b, d)$ or a control sample of thermally fibrillized collagen (e). The scale bar in (a) indicates $50 \mu \mathrm{m}$ for panels (a,b) while the scale bar in (c) indicates $50 \mu \mathrm{m}$ for panels (c,d,e). In (e), "m" denotes the fibrillar collagen control matrix. 

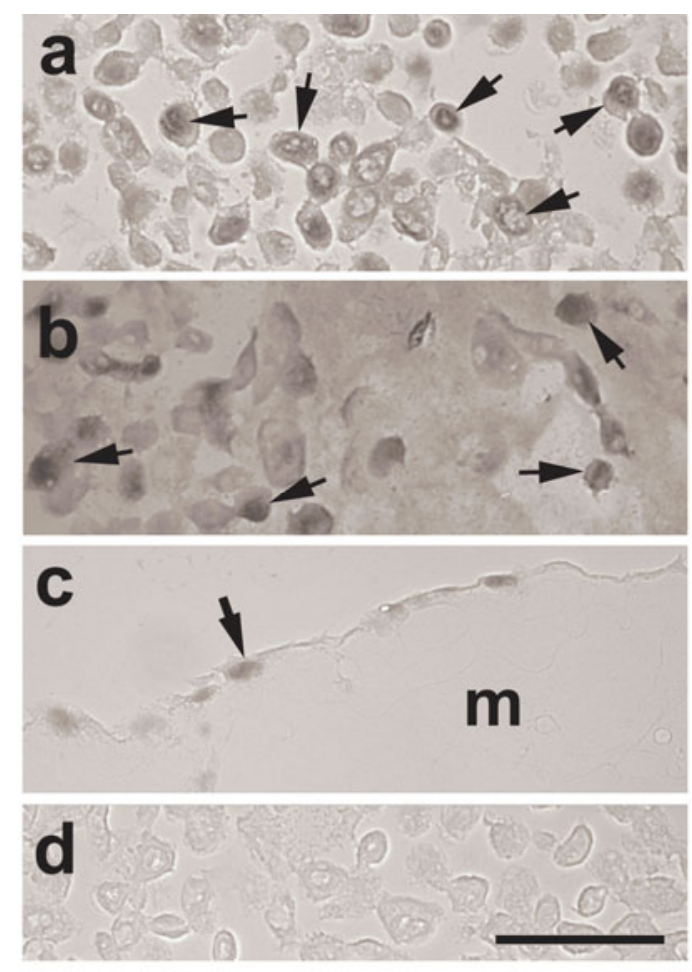

Figure 3. Cell proliferation marker expression differences, when staining for PCNA, are indicated by the contrast among (a) punctate nucleoplasmal staining in plastic-replated cells, (b) punctate nucleoplasmal staining on control thermally fibrilized collagen, and (c) the diffuse staining for cells replated on the electrochemically produced matrix. In (c), "m" denotes the matrix. Negative control immunostaining reactions (shown in (d) for plasticreplated cells) confirm the specificity of the anti-PCNA stain. The scale bar indicates $50 \mu \mathrm{m}$ in (a-d). 


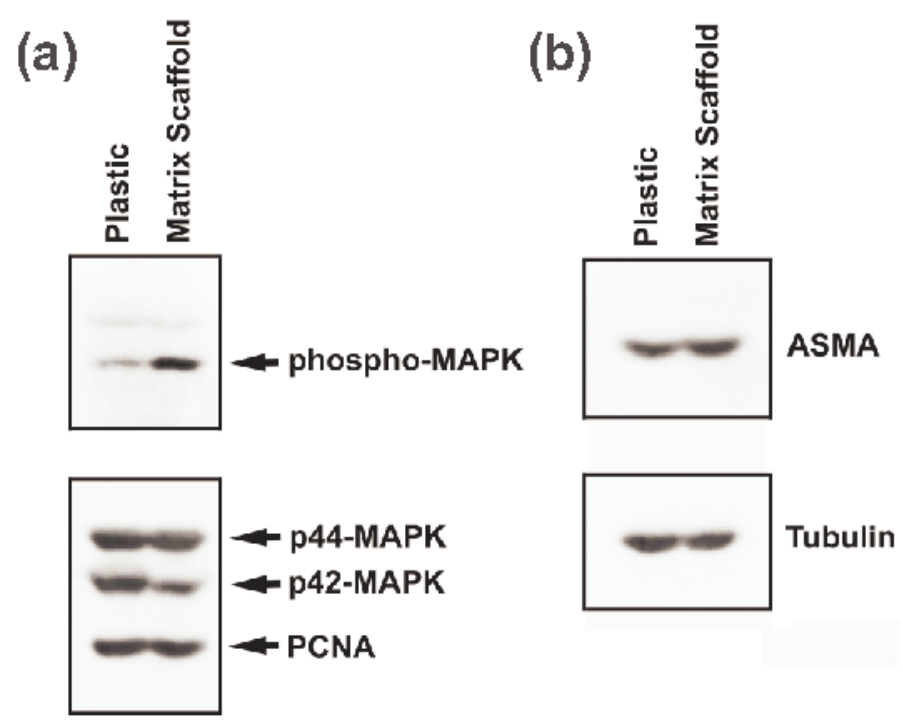

Figure 4. Western blot analysis of (a) PCNA protein expression, phospho-MAPK (phosphop42 and phospho-p44), total MAPK (p42 and p44), and (b) ASMA, tubulin of either plasticreplated or electrochemical matrix-replated MK/T-1 cells. Densitometry analysis of western blot data reveal a slight but significant upregulation of ASMA expression in the electrochemical matrix replated cultures and a significant upregulation of p42-phosho-MAPK expression in electrochemical matrix replated cultures. 


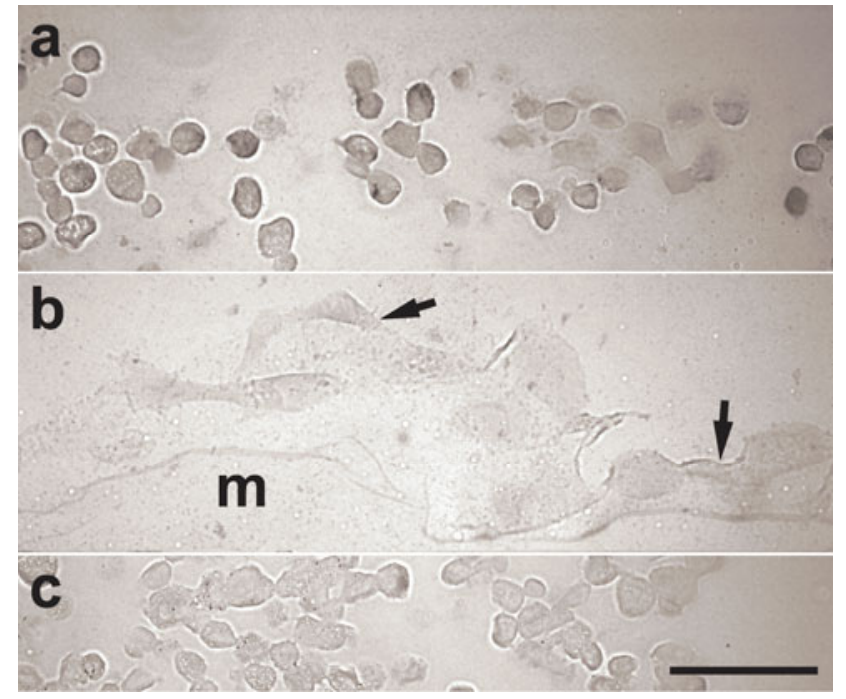

Figure 5. The contrast between strong staining for BrdU in (a) plastic-replated cells and (b) the relative lack of BrdU staining for electrochemical matrix-replated cells (where "m" denotes the matrix) indicates a decrease in proliferative response of the MK/T-1 cells on the matrix. Negative control immunostaining reactions (shown in (c) for plastic-replated cells) confirm the specificity of the BrdU stain. The scale bar shows $50 \mu \mathrm{m}$ for (a-c). 

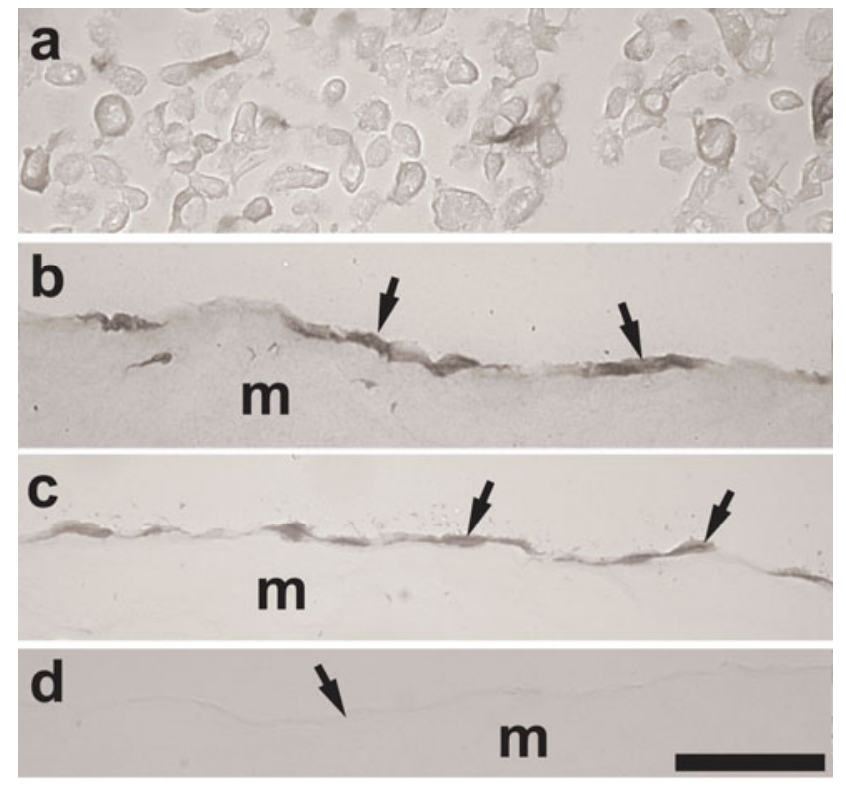

Figure 6. Cell signaling is confirmed by retention of stain for ASMA in cells (a) replated onto tissue culture plastic and (b) replated onto the electrochemical collagen matrix. Evidence of robust phospho-MAPK (phospho-p42 and phospho-p44) staining (c) and cell morphologies in $H \& E$ counterstains in adjacent sections of the matrix (not shown) provide further positive controls for cell signaling activity and cellular integrity. (d) Negative control immunostaining reactions confirm the specificity of the ASMA stain. The scale bar indicates $50 \mu \mathrm{m}$ in all panels, and "m" denotes the matrix. 
A transparent, electrochemically produced collagen matrix shows promise as a scaffold for cornea repair. Immortalized corneal fibroblasts seeded onto this matrix are viable but do not proliferate, similar to their behavior in a native corneal environment. This unusual matrix property - the ability to contain cell growth - could also be relevant for controlling cell growth in engineered tissues.

Robert Gendron*, M. Ramesh Kumar, Helene Paradis*, Darryl Martin, Nhu Ho, Danielle Gardiner, Erika F. Merschrod S.*, Kristin M. Poduska*

Controlled cell proliferation on an electrochemically engineered collagen scaffold

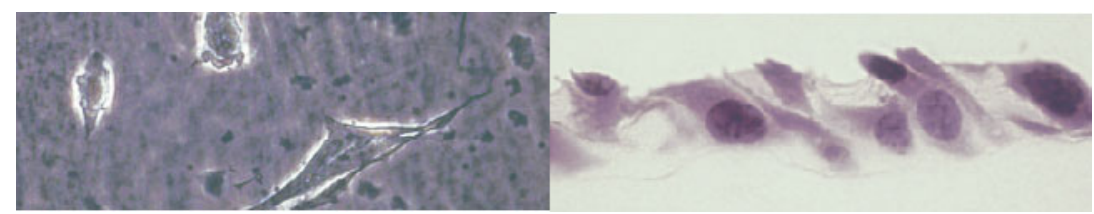

